\title{
Immunotherapy beyond cellular therapy in Follicular Lymphoma: A case of complete remission after failure of two CAR-T
}

\author{
Adam Kase $^{1}$, Mohamed Kharfan Dabaja ${ }^{2}$, Andrew Donaldson ${ }^{1}$, Jamie Elliott ${ }^{1}$, and \\ Taimur Sher ${ }^{1}$ \\ ${ }^{1}$ Mayo Clinic in Florida \\ ${ }^{2}$ Mayo Clinic Hospital Jacksonville
}

January 30, 2022

\begin{abstract}
Patients with relapsed follicular lymphoma who do not respond to CAR-T have a poor outcome. We present a case of refractory follicular lymphoma who relapsed after two CAR-T infusions and achieved a complete remission after treatment with obinutuzumab and lenalidomide. This represents a promising treatment option in the post-CAR-T setting.

Immunotherapy beyond cellular therapy in Follicular Lymphoma: A case of complete remission after failure of two CAR-T

Adam M. Kase, M.D. ${ }^{1}$, Mohamed A. Kharfan-Dabaja, M.D. ${ }^{2}$, Andrew Donaldson R.N. ${ }^{1}$, Jamie Elliott APRN, D.N.P. ${ }^{1}$, Taimur Sher, M.B.B.S, M.D. ${ }^{1}$

${ }^{1}$ Mayo Clinic Florida Division of Hematology Oncology

${ }^{2}$ Mayo Clinic Florida Division of Hematology Oncology, Blood and Marrow Transplantation and Cellular Therapies Program

Adam Kase: kase.adam@mayo.edu, Mohamed Kharfan-Dabaja: kharfandabaja.mohamed@mayo.edu, Andrew Donaldson: Donaldson.andrew@mayo.edu, Jamie Elliott: Elliott.jamie@mayo.edu, Taimur Sher: sher.taimur@mayo.edu

Data Availability: The data that support the findings of this study are not publicly available due to privacy or ethical restrictions.

The authors have no conflicts of interests.

Patient consent was obtained for the publication of this case report.

No funding was provided for this manuscript.

Introduction:

Follicular lymphoma is the most common indolent non-Hodgkin lymphoma which is known for its relapsing-remitting disease course with $20 \%$ of patients developing progression of disease within 2 years of chemoimmunotherapy. ${ }^{1}$ The treatment for relapsed follicular lymphoma includes combinations of chemoimmunotherapy, immune modulators and hematopoietic stem cell transplant. More recently chimeric antigen receptor T-cell therapy (CAR-T) has demonstrated impressive activity in the relapsed/refractory setting leading to the FDA approval of axicabtagene ciloleucel in February 2021 for relapsed or refractory follicular lymphoma. ${ }^{2}$ Patients not responding to CAR-T or relapsing soon after treatment have a poor outcome.
\end{abstract}


3 The optimal treatment for such CAR-T treatment failure remains unclear. Here we present a case of relapse refractory follicular lymphoma who relapsed after multiple lines of chemoimmunotherapy including two CAR-T infusions, who then achieved a complete remission after treatment with obinutuzumab and lenalidomide.

Case Report:

The patient is a 73-year-old female who was diagnosed with stage IVA follicular lymphoma in 2000. Over a period of 20 years, she received the following treatments with variable responses (Table 1), including: cyclophosphamide, vincristine and prednisone, followed by multiple rounds of rituximab, targeted radiation, a clinical trial with everolimus, single agent lenalidomide, an oral PI3K delta inhibitor on a clinical trial and rituximab plus gemcitabine with oxaliplatin. Despite all these therapies she continued to progress. She was enrolled in ZUMA-5 clinical trial for treatment with axicabtagene ciloleucel CAR-T and obtained a complete remission that lasted for approximately 1.5 years. At relapse, she underwent a second CD19 CAR-T in June 2020 and had florid progression of disease just two months later in August 2020 (Figure 1A). She briefly enrolled on a clinical trial with a novel BCL-2 inhibitor but had rapid disease progression within few days. In October 2020, she was started on obinutuzumab and lenalidomide. The dose of lenalidomide had to be reduced from $20 \mathrm{mg}$ (days 1-21) to $5 \mathrm{mg}$ (days 1-14) due to thrombocytopenia. A PET scan was obtained to assess response to treatment after 2 months and revealed radiologic complete remission. Ongoing surveillance PET scan (Figure 1B) in April 2021 showed continued remission, consistent with a 6 -month durable radiologic complete remission after 5 cycles of obinutuzumab and lenalidomide two weeks on one week off.

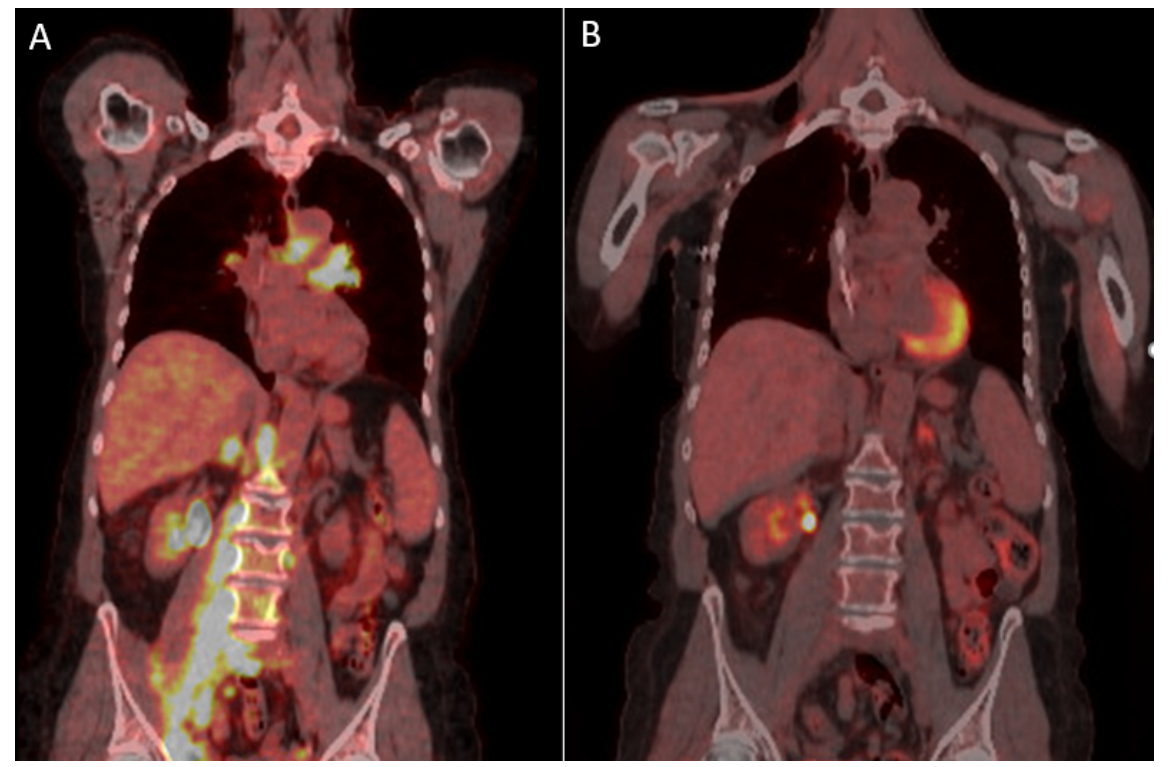

Figure 1: (A) FDG-PET scan demonstrating extensive hypermetabolic soft tissue activity involving the mediastinal bilateral hilar node chains with extensive confluent mesenteric, retroperitoneal, pelvic and inguinal lymph node disease. (B) Post treatment FDG-PET demonstrating complete remission.

\begin{tabular}{lll}
\hline Treatment & Duration of Treatment & Progression Free Survival (mon \\
\hline CVP & 6 cycles & 16 \\
Rituximab (start $\mathbf{8 / 9 / 2 0 0 1 )}$ & 8 cycles & 31 \\
Rituximab (start $\mathbf{4 / 2 0 0 4 )}$ & 5 cycles & 15 \\
Radiation Therapy & 2000 cGy in 10 fractions & 1
\end{tabular}




\begin{tabular}{lll}
\hline Treatment & Duration of Treatment & Progression Free Survival (mon \\
\hline Everolimus trial (start 1/2007) & 12 months & 12 \\
Lenalidomide start 9/27/2010) & 5 months & 5 \\
Rituximab (start 2/2011) & 8 cycles & 11 \\
Lenalidomide (start 1/2012) & 28 months & 28 \\
PI3K delta Inhibitor trial (6/2014) & 6 months & 6 \\
Gemcitabine and Oxaliplatin (start 12/30/2014) & 7 cycles & 19 \\
Axicabtagene ciloleucel (9/2018) & N/A & 20 \\
Axicabtagene ciloleucel (6/2020) & N/A & 2 \\
BCL-2 inhibitor trial & 2 weeks & 0 \\
Obinutuzumab and Lenalidomide & 5 cycles & Ongoing $(>6)$ \\
Lenalidomide maintenance & Ongoing & Ongoing $(>6)$ \\
\hline
\end{tabular}

Table 1: Treatment history demonstrating duration of treatment, progression free survival and best treatment response.

Discussion:

Relapsed follicular lymphoma can be treated with a variety of modalities including chemoimmunotherapy, immune modulators, hematopoietic stem cell transplant and CAR-T (Table 2). Rituximab has been studied in heavily pretreated and in patients who had previously responded to rituximab and resulted in overall response rate (ORR) $40 \%$ and complete response (CR) in $11 \%$ of patients. ${ }^{4}$ Chemotherapy can also be added to monoclonal antibodies in patients with untreated and relapsed follicular lymphoma to achieve an improved overall survival compared to patients with chemotherapy alone. ${ }^{5}$ In the GADOLIN trial, rituximab refractory indolent non-Hodgkin lymphoma was treated with obinutuzumab plus bendamustine. In the intention-totreat group the median progression-free survival was 25.8 months and 14.1 months, in the combination and monotherapy arms respectively. ${ }^{6}$ Combination was given for 6 cycles followed by maintenance obinutuzumab every 2 months for 2 years until progression. Immune modulators have also been incorporated into these regimens. Lenalidomide can be combined with rituximab or obinutuzumab for early or late relapse. In the AUGMENT trial, patients received lenalidomide or placebo for 12 cycles plus rituximab weekly. ${ }^{7}$ The median PFS was improved from 14 months to 39 months. Obinutuzumab was combined with lenalidomide after rituximab-containing therapy in a phase Ib trial with $63 \%$ of patients achieving a response, as seen in this patient. ${ }^{8}$ While these regimens have a higher toxicity profile, combining these agents in patients with a good performance status appears to be beneficial.

\begin{tabular}{|c|c|c|c|}
\hline Regimen & Overview & $\begin{array}{l}\text { Overall Response } \\
\text { Rate }\end{array}$ & Survival Outcomes \\
\hline $\begin{array}{l}\text { Single agent } \\
\text { Rituximab }^{4}\end{array}$ & $\begin{array}{l}\text { Low tumor burden, poor } \\
\text { performance status, } \\
\text { slowly progressive }\end{array}$ & $\begin{array}{l}66 \% \text { (initial) } 40 \% \\
\text { (re-treatment) }\end{array}$ & $\begin{array}{l}\text { mPFS: } 21 \text { months } \\
\text { (initial) mPFS: } 18 \\
\text { months (re-treatment) }\end{array}$ \\
\hline $\begin{array}{l}\text { Rituximab }+ \\
\text { Chemo }^{5}\end{array}$ & $\begin{array}{l}\text { First relapse, or late } \\
\text { relapse }\end{array}$ & $\begin{array}{l}\text { RR } 1.28 \text { favoring } \\
\text { R-chemo }\end{array}$ & $\begin{array}{l}\text { HR for mortality: } 0.632 \mathrm{y} \\
\text { OS: } 90 \%\end{array}$ \\
\hline Obinutuzumab $+\mathrm{Be}$ & aRsituixénab refractory & $57 \%$ & mPFS: 25.8 months \\
\hline $\begin{array}{l}\text { Anti-CD20 + } \\
\text { lenalidomide }^{7,9}\end{array}$ & Late or early relapse & $65-77 \%$ & $\begin{array}{l}\text { mPFS: } 39 \text { months } \\
\text { mTTP: } 24 \text { months }\end{array}$ \\
\hline Lenalidomide $^{9}$ & Late or early relapse & $53 \%$ & mTTP: 13.2 months \\
\hline Idelalisib $^{10}$ & $\begin{array}{l}\text { After two prior } \\
\text { therapies }\end{array}$ & $57 \%$ & mPFS: 11 months \\
\hline Tazemetostat $^{11}$ & $\begin{array}{l}\text { EZH2 mutation in first } \\
\text { relapse }\end{array}$ & $69 \%$ & mPFS: 11 months \\
\hline Autologous HSCT ${ }^{12}$ & Early treatment failure & $78 \%$ & $5 y$ OS $73 \%$ \\
\hline
\end{tabular}




\begin{tabular}{llll}
\hline Regimen & Overview & $\begin{array}{l}\text { Overall Response } \\
\text { Rate }\end{array}$ & Survival Outcomes \\
\hline $\begin{array}{l}\text { Axicabtagene } \\
\text { ciloleucel }^{\mathbf{2}}\end{array}$ & $\begin{array}{l}\text { After two prior } \\
\text { therapies }\end{array}$ & $95 \%$ & 12 month DOR: $71.7 \%$ \\
$\begin{array}{l}\text { Pembrolizumab }++{ }^{\mathbf{1 3}} \\
\text { Lenalidomide } \\
+/ \text {-anti-CD20 }++^{\mathbf{1 4}}\end{array}$ & $\begin{array}{l}\text { After failed CAR-T } \\
\text { After failed CAR-T }\end{array}$ & $\begin{array}{l}27 \% \\
63.6 \% \text { (within 15 days of } \\
\end{array}$ & $\begin{array}{l}\text { CAR-T infusion) } 18.8 \% \\
\text { (15 days after CAR-T }\end{array}$ \\
& & infusion) & \\
\hline
\end{tabular}

Table 2: Response rates and outcomes for the treatment of relapsed or refractory follicular lymphoma.

+CHOP, CNOP, CVP, FCM, MCP. ++ Includes multiple subtypes of lymphoma including DLBCL, primary B-cell lymphoma, transformed follicular. mPFS, median progression free survival; HR, hazard ratio; mTTP, time to progression; DOR, duration of response; n/a, not available.

The recent approval of axicabtagene ciloleucel for the treatment of relapsed or refractory follicular lymphoma after two prior lines of therapy was based on the results of a ZUMA-5 which demonstrated $80 \%$ complete response rate and 12 month durable response of $72 \%{ }^{2}$ This offers an effective treatment option for patients who develop refractory disease. However, treatment after disease progression following CAR-T is unclear and provides an opportunity for further investigation. With the CAR-T FDA approval occurring after two lines of treatment, patients with relapsed follicular lymphoma will be receiving this therapeutic modality earlier in their disease course which opens the door for re-treatment with previous used regimens or unused combinations guided by mechanisms of treatment failure. The mechanisms of CAR-T treatment failure have been divided into three categories including tumor intrinsic factors (i.e. loss of CD19 epitope), other host factors (i.e. negative regulatory pathways, high tumor burden) and inadequacies of the CAR-T cell. ${ }^{15}$ Clinical trials are ongoing to overcome these mechanisms of treatment failures including targeting programmed death-ligand $1 /$ programmed cell death protein 1 . A phase I/II trial is underway using pembrolizumab in patients failing to respond to CD19 CAR-T or relapse after CAR-T. ${ }^{13}$ Of the 11 patients, 2 patients had partial response and $1 \mathrm{CR}$ suggesting benefit that could be the result of altering CAR T-cell exhaustion, or immunosuppressive tumor microenvironment.

Lenalidomide has been investigated for the treatment of lymphoma based on its direct antineoplastic effects on malignant B-cells, and cytotoxicity mediated by T cells and NK cells. ${ }^{16}$ The malignant B-cell effects of lenalidomide are shown through its ability to regulate cyclin dependent kinases decreasing cellular proliferation, down regulation of programmed death ligand-1, and AKT inhibition all resulting in antineoplastic and antiproliferation. Lenalidomide combats cancers ability to evade the immune system, a key defensive mechanism. While lymphoma cells induce impaired immune synapse formation and effector function impacting antigen presentation, lenalidomide has been shown to repair this immune synapse formation in an Ex vivo model, ${ }^{17}$ thereby enhancing $\mathrm{T}$ cell mediated cytotoxicity. Additional improvement in cellular cytotoxicity can be seen with lenalidomide's impact on NK cell activity. In patients treated with lenalidomide, NK cell number and activity were increased resulting in enhanced antibody dependent cellular cytotoxicity (ADCC) and NK cell induced cytotoxicity. ${ }^{18}$ With understanding the mechanisms of action, lenalidomide has been combined with other agents to achieve synergistic activity. When the anti-CD20 monoclonal antibody, rituximab, is used as an antineoplastic agent it results in the direct induction of apoptosis and ADCC. When lenalidomide is combined with rituximab it results in enhanced apoptosis via upregulation of c-Jun N-terminal protein kinase phosphorylation and activating the mitochondrial derived apoptotic pathway. ${ }^{19}$ This combined therapy was performed in mantle cell lymphoma mouse models and resulted in synergistic activity causing decrease tumor burden by two fold and improved survival time compared to single agents. ${ }^{19}$

Lenalidomide success in the post-CAR-T setting has been demonstrated in a small cohort of patients presented at the 2020 American Society of Hematology by Dr. Thieblemont et al. Eleven patients with varying 
lymphoma subtypes who progressed within 15 days post-CD19 CAR-T infusion were treated with lenalidomide with or without rituximab or with obinutuzumab. ${ }^{14}$ An ORR of $63.6 \%$ was observed with a CR of $36.4 \%$. Response rates with lenalidomide were also seen in patients who progressed/relapsed after 15 days from CAR-T infusion with an ORR of $18.8 \%$ and CR of $10.4 \%$. In addition to response, patient's receiving lenalidomide also had a higher CAR-T expansion in the blood. These results with lenalidomide are promising based on its antitumor response and immunomodulatory impact, mechanisms which are necessary as patient's progress after CAR-T. The response rate in this cohort as well as our case presented here is likely the combined result of the synergistic activity of lenalidomide plus anti-CD20 monoclonal antibody in addition to lenalidomides immune modulatory effects which aid in cellular cytotoxicity and achieving a durable response.

Conclusion:

The complete remission seen in this patient suggests the use of lenalidomide with obinutuzumab could be used in follicular lymphoma in the post-CAR-T setting, analogous to published data with other lymphoma subtypes. With CAR-T being an approved third line option for follicular lymphoma, further research is warranted to determine appropriate sequencing of agents leading up to CAR-T and post-CAR-T failure in order to optimize survival outcomes. With lenalidomide's T-cell and antitumor effects, begs the question of where this agent should be in the sequence of treatments? Whether it is included as therapy pre-CAR-T, maintenance post CAR- $\mathrm{T}$, or salvage post CAR-T, still requires further investigation. While confirmation in a large clinical trial is needed, lenalidomide plus obinutuzumab could be considered as a treatment option in patients with follicular lymphoma who progress/relapse post CAR-T.

\section{References}

1. Casulo C, Barr PM. How I treat early-relapsing follicular lymphoma. Blood 2019;133:1540-7.

2. Jacobson C, Chavez JC, Sehgal AR, et al. Primary Analysis of Zuma-5: A Phase 2 Study of Axicabtagene Ciloleucel (Axi-Cel) in Patients with Relapsed/Refractory (R/R) Indolent Non-Hodgkin Lymphoma (iNHL). Blood 2020;136:40-1.

3. Chow VA, Gopal AK, Maloney DG, et al. Outcomes of patients with large B-cell lymphomas and progressive disease following CD19-specific CAR T-cell therapy. Am J Hematol 2019;94:E209-e13.

4. Davis TA, Grillo-López AJ, White CA, et al. Rituximab anti-CD20 monoclonal antibody therapy in non-Hodgkin's lymphoma: safety and efficacy of re-treatment. J Clin Oncol 2000;18:3135-43.

5. Schulz H, Bohlius JF, Trelle S, et al. Immunochemotherapy with rituximab and overall survival in patients with indolent or mantle cell lymphoma: a systematic review and meta-analysis. J Natl Cancer Inst 2007;99:706-14.

6. Cheson BD, Chua N, Mayer J, et al. Overall Survival Benefit in Patients With Rituximab-Refractory Indolent Non-Hodgkin Lymphoma Who Received Obinutuzumab Plus Bendamustine Induction and Obinutuzumab Maintenance in the GADOLIN Study. J Clin Oncol 2018;36:2259-66.

7. Leonard JP, Trneny M, Izutsu K, et al. AUGMENT: A Phase III Study of Lenalidomide Plus Rituximab Versus Placebo Plus Rituximab in Relapsed or Refractory Indolent Lymphoma. J Clin Oncol 2019;37:118899.

8. Morschhauser F, Salles G, Le Gouill S, et al. An open-label phase 1b study of obinutuzumab plus lenalidomide in relapsed/refractory follicular B-cell lymphoma. Blood 2018;132:1486-94.

9. Leonard JP, Jung SH, Johnson J, et al. Randomized Trial of Lenalidomide Alone Versus Lenalidomide Plus Rituximab in Patients With Recurrent Follicular Lymphoma: CALGB 50401 (Alliance). J Clin Oncol 2015;33:3635-40.

10. Gopal AK, Kahl BS, de Vos S, et al. PI3Kס inhibition by idelalisib in patients with relapsed indolent lymphoma. N Engl J Med 2014;370:1008-18. 
11. Morschhauser F, Tilly H, Chaidos A, et al. Tazemetostat for patients with relapsed or refractory follicular lymphoma: an open-label, single-arm, multicentre, phase 2 trial. Lancet Oncol 2020;21:1433-42.

12. Casulo C, Friedberg JW, Ahn KW, et al. Autologous Transplantation in Follicular Lymphoma with Early Therapy Failure: A National LymphoCare Study and Center for International Blood and Marrow Transplant Research Analysis. Biology of blood and marrow transplantation : journal of the American Society for Blood and Marrow Transplantation 2018;24:1163-71.

13. Chong EA, Svoboda J, Dwivedy Nasta S, et al. Sequential Anti-CD19 Directed Chimeric Antigen Receptor Modified T-Cell Therapy (CART19) and PD-1 Blockade with Pembrolizumab in Patients with Relapsed or Refractory B-Cell Non-Hodgkin Lymphomas. Blood 2018;132:4198-.

14. Thieblemont C, Sylvie Chevret, Vincent Allain, Roberta Di Blasi, Florence Morin, Laetitia Vercellino, Sandrine Roulland, Karin Tarte, Veronique Meignin, Sophie Caillat-Zucman. Lenalidomide Enhance CAR T-Cells Response in Patients with Refractory/Relapsed Large B-Cell Lymphoma Experiencing Progression after Infusion. 62nd American Society of Hematology Annual Meeting and Exposition 2020;Abstract 1115.

15. Byrne M, Oluwole OO, Savani B, Majhail NS, Hill BT, Locke FL. Understanding and Managing Large B Cell Lymphoma Relapses after Chimeric Antigen Receptor T Cell Therapy. Biology of blood and marrow transplantation : journal of the American Society for Blood and Marrow Transplantation 2019;25:e344-e51.

16. Gribben JG, Fowler N, Morschhauser F. Mechanisms of Action of Lenalidomide in B-Cell Non-Hodgkin Lymphoma. Journal of clinical oncology : official journal of the American Society of Clinical Oncology 2015;33:2803-11.

17. Ramsay AG, Clear AJ, Fatah R, Gribben JG. Multiple inhibitory ligands induce impaired T-cell immunologic synapse function in chronic lymphocytic leukemia that can be blocked with lenalidomide: establishing a reversible immune evasion mechanism in human cancer. Blood 2012;120:1412-21.

18. Hayashi T, Hideshima T, Akiyama M, et al. Molecular mechanisms whereby immunomodulatory drugs activate natural killer cells: clinical application. Br J Haematol 2005;128:192-203.

19. Zhang L, Qian Z, Cai Z, et al. Synergistic antitumor effects of lenalidomide and rituximab on mantle cell lymphoma in vitro and in vivo. Am J Hematol 2009;84:553-9. 